\title{
A cosmeceutical formulation based on boswellic acids for the treatment of erythematous eczema and psoriasis
}

\author{
Stefano Togni' \\ Giada Maramaldi ${ }^{\prime}$ \\ Francesco Di Pierro ${ }^{2}$ \\ Massimo Biondi ${ }^{3}$ \\ 'Indena S.p.A., Milan, Italy; ${ }^{2}$ Velleja \\ Research, Milan, Italy; ${ }^{3}$ Dermatology \\ Unit, ASL Piacenza, Piacenza, Italy
}

Correspondence: Giada Maramaldi Indena S.p.A, Viale Ortles I2-20I39, Milan, Italy

Tel/Fax +3902574963I5

Email giada.maramaldi@indena.com
This article was published in the following Dove Press journal:

Clinical, Cosmetic and Investigational Dermatology

II November 2014

Number of times this article has been viewed

Background: Boswellic acids (BAs) show anti-inflammatory properties in a variety of inflammatory diseases, including rheumatoid arthritis, osteoarthritis, and asthma. A topical administration route is currently used to deliver active compounds in psoriatic and eczematous patients. In this double-blind study we compare a novel BA formulation (containing Bosexil ${ }^{\circledR}$, INCI [International Nomenclature of Cosmetic Ingredients]: lecithin, Boswellia serrata resin extract) with a placebo formulation. A third arm of the trial received a formulation of Vaccinium myrtillus seed oil, previously demonstrated as an effective local treatment for psoriatic lesions.

Methods: Patients with psoriasis or erythematous eczema were randomly assigned, in a 1:1:1 ratio, to Bosexil ${ }^{\circledR}$, V. myrtillus seed oil, or placebo. In order to evaluate the effects of treatment, the changes of scales and erythema from diagnosis to the end of treatment were scored in psoriatic patients, while changes in itch and erythema were analyzed for erythematous eczema patients. Psoriasis Area Severity Index and Eczema Area and Severity Index scores were also calculated.

Results: In patients with psoriasis, scales and erythema improved both with Bosexil ${ }^{\circledR}$ and the V. myrtillus seed oil treatment in comparison with placebo. In particular, the treatment with Bosexil $^{\circledR}$ formulation improved scales ( $70 \%$ of cases) and erythema (50\% of cases) without any case of worsening. In patients with eczema, the administration of placebo did not result in any improvement in $90 \%$ of cases, and in the remaining $10 \%$ worsened both itch and erythema. Bosexil ${ }^{\circledR}$ formulation improved both itch ( $60 \%$ of cases) and erythema ( $60 \%$ of cases) without any case of worsening. V. myrtillus seed oil improved itch and erythema in $66.7 \%$ and $77.8 \%$ of patients, respectively.

Conclusion: A topical formulation of Bosexil ${ }^{\mathbb{B}}$ may be promising for the treatment of psoriasis and erythematous eczema. Long-term studies are recommended to evaluate the adherence to this topical treatment and its clinical benefits in real life.

Keywords: boswellic acids, cosmeceutical, inflammation, psoriasis, erythematous eczema

\section{Introduction}

Among dermatological disorders, erythematous dermatitis, or eczema, and psoriasis are the most common. ${ }^{1}$ Dermatitis is defined as a superficial skin inflammation, characterized by poorly delimited erythema, and usually itchy crusts and scales. ${ }^{2}$ Conventional measures to prevent eczema include avoidance of irritants and potential allergens, as well as continued hydration of the skin with thick emollients. Topical corticosteroids and topical immune modulators are often used as a primary treatment. Other therapies including phototherapy, antimicrobials, antihistamines, and systemic immunosuppressives are also therapeutic options. ${ }^{1}$ 
Psoriasis is an autoimmune, chronic, noncontagious, recurrent, inflammatory skin disorder. In Western populations, its prevalence is estimated to be as high as $2.8 \%{ }^{3}$ The typical skin lesions of psoriatic patients are sharply demarcated erythematous plaques covered with silvery or opalescent scales. ${ }^{4}$ The inflammatory response is supported by the infiltration of polymorphic neutrophils in the skin. Neutrophils are triggered by the chemokines and lymphokines released by keratinocytes and T-lymphocytes, respectively, and, in turn, trigger the activity of lymphocytes and keratinocytes, thus generating a vicious cycle limited to acute lesions but linked in any case to the extended inflammation. ${ }^{5}$ Treatments for psoriasis include immunosuppressive drugs such as methotrexate, cyclosporine, and fumaric acid esters. ${ }^{6}$ However, advances in mechanistic understanding of signaling pathways involved in the pathogenesis of psoriasis have led to the testing of biological therapies. These include immune suppressive drugs (eg, alefacept) and anticytokine therapies (antitumor necrosis factor [TNF] therapies [adalimumab, etanercept, infliximab, ustekinumab]). ${ }^{7}$

Topical application of anti-inflammatory molecules is a feasible alternative to systemic approaches to treat psoriatic and eczematous symptoms by directly acting on inflammatory processes and generating a marked soothing response in skin. ${ }^{8}$ A recent study on costs of psoriasis medications has demonstrated that biological treatments represent $67 \%$ of total medication costs, and only $5 \%$ of patients purchase them. In contrast, topical medications are the most commonly purchased treatments and account for the $18 \%$ of total costs. ${ }^{9}$ Furthermore, since almost $10 \%$ of patients require at least three topical therapy switches over a 1-year period to gain health benefit, the identification and formulation of novel anti-inflammatory compounds that could be used in topical application are a medical need for psoriasis treatment and a possible approach to reduce costs. ${ }^{9}$

Boswellia serrata gum resin extracts (BSEs) (or olibanum) are used in traditional ayurvedic medicine to treat inflammatory diseases. The prevalent component of lipophilic fraction of olibanum is represented by the pentacyclic triterpenes boswellic acids (BAs), several forms of which have been characterized by analytical techniques, including $\alpha$ - and $\beta$-configured BAs (Figure 1). Another structural variety is given by the presence of a carbonyl group and an acetyl group in 11-keto BAs (KBA) and 3-O-acetyl-BAs (AKBA), respectively. ${ }^{10}$ Animal studies and pilot clinical trials support the efficacy of BSEs for the treatment of a variety of inflammatory diseases such as inflammatory bowel disease, rheumatoid arthritis, osteoarthritis, and asthma. ${ }^{11-14}$ In 2002, the European Medicines Agency classified BSE as an "orphan drug" for the treatment of peritumoral brain edema. ${ }^{15}$ The main anti-inflammatory properties of BAs are attributed to suppression of leukotriene formation via inhibition of 5-lipoxygenase (5-LO) by KBA and AKBA. ${ }^{15}$ The mechanistic model has to be fully elucidated, but there is some evidence of an indirect action of BAs leading to an irreversible inhibition of 5-LO by a $\mathrm{Ca} 2+-$ mediated pathway. ${ }^{16}$ BAs also inhibit the human leukocyte elastase, released in inflammation- and hypersensitivity-based human disorders, thus further supporting BA's antiphlogistic properties. ${ }^{17}$ In activated human monocytes, AKBA conveys inhibition of nuclear factor (NF)-kB and subsequent downregulation of TNF- $\alpha$ expression, via its direct inhibition of I kappa B kinase (IKK) ${ }^{18}$ Noteworthy is that when the IKK inhibition by BAs has been investigated in the CD18 hypomorphic (CD18[hypo]) mouse model of psoriasis, the suppressive effects on NF-kB signaling have been confirmed, thus suggesting that targeting NF-kB with BAs may be an effective strategy for the treatment of psoriasis. ${ }^{19}$

Since both psoriasis and erythematous dermatitis present a prevalent inflammatory component and BAs have a documented inhibitory activity in some pathways with a major role in inflammation, especially in NF-kB pathway, we developed a formulation containing BAs with potential efficacy in patients with psoriasis and erythematous dermatitis. In this
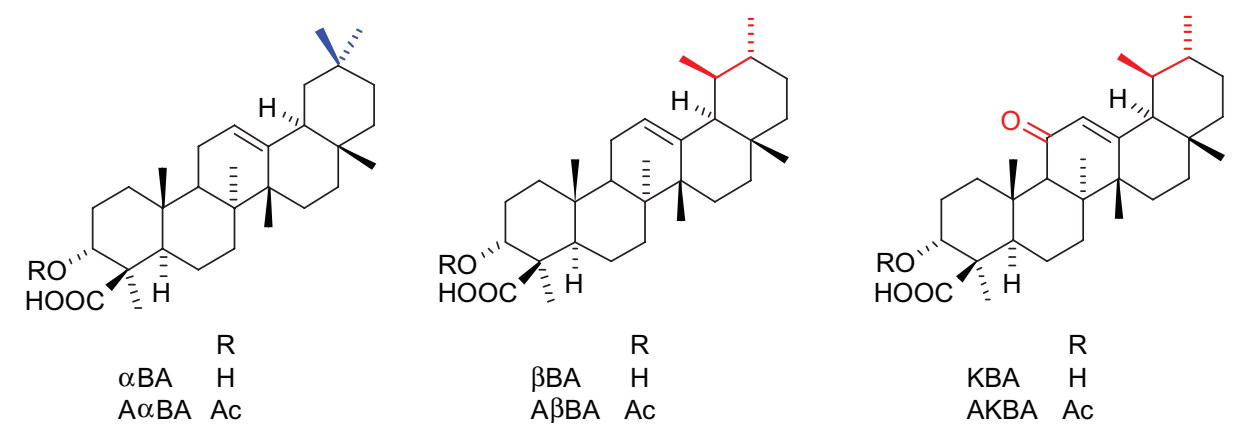

Figure I Boswellic acids structures. 
double-blind study, we compare, for the first time, a novel BA formulation (containing Bosexil ${ }^{\circledR}$, INCI: lecithin, BSE) with a placebo formulation in the treatment of psoriasis or eczema. A third arm of the trial received a formulation of Vaccinium myrtillus seed oil, which was previously demonstrated as an efficient local treatment for psoriatic lesions. ${ }^{20}$

\section{Patients and methods}

This is a randomized, placebo-controlled, double-blind study performed at the Department of Dermatology at the University of Varese (Italy) in collaboration with Velleja Research and the Local Health Agency 1 (ASL1) in the Piacenza (Italy) area. We separately considered in the study patients who were affected either by psoriasis or by erythematous dermatitis. The diagnosis of erythematous atopic eczema or psoriasis was made by the physician in charge of the Department of Dermatology involved in the trial. All patients signed an informed consent. Patients had to be free from any topical or systemic anti-inflammatory treatment for at least 3 months.

Patients were randomly assigned, in a 1:1:1 ratio and according to a computer-generated numerical table, to one of the following groups: Bosexil ${ }^{\circledR}, V$. myrtillus seed oil, or placebo. No crossovers were allowed during the study period. All formulations were topically applied (cream) and were commercially available. Their compositions are reported to be as shown in Tables 1-3.

Bosexil $^{\circledR}$ was formulated according to the Phytosome ${ }^{\circledR}$ delivery system that is aimed at improving the bioavailability and skin affinity of bioactive molecules. ${ }^{21}$

All preparations could not be distinguished without a chemical analysis. Patients were asked to apply the cream twice a day to the affected areas in a sufficient quantity to cover the whole skin area involved, for a total period of 30 days. Other concomitant pharmacological treatments were not interrupted or modified, except for those, dermatological or oral, finalized to treat psoriasis.

Table I Composition of Bosexil ${ }^{\circledR}$

\begin{tabular}{ll}
\hline Aqua (water) & 82.90 \\
Glycerin & 2.00 \\
Lecithin, Boswellia serrata resin extract $\left(\right.$ Bosexi $\left.^{\circledR}\right)$ & 0.50 \\
Disodium ethylenediaminetetraacetic acid & 0.10 \\
Imidazolidinyl urea & 0.30 \\
Polyacrylamide, CI3-I4 Isoparaffin, Laureth-7 & 3.00 \\
Hydrogenated polydecene & 8.50 \\
Caprylic/capric triglyceride & 2.00 \\
Lecithin, tocopherol, ascorbyl palmitate, citric acid & 0.10 \\
Phenoxyethanol & 0.60 \\
\hline
\end{tabular}

Note: All values are represented as percentages.
Table 2 Composition of Vaccinium myrtillus seed oil

\begin{tabular}{ll}
\hline Aqua (water) & 83.40 \\
Glycerin & 2.00 \\
Disodium ethylenediaminetetraacetic acid & 0.10 \\
Imidazolidinyl urea & 0.30 \\
Polyacrylamide, CI3-I4 Isoparaffin, Laureth-7 & 3.00 \\
Hydrogenated polydecene & 6.50 \\
Caprylic/capric triglyceride & 2.00 \\
Vaccinium myrtillus seed oil (Omegablue ${ }^{\circledR}$ ) & 2.00 \\
Lecithin, tocopherol, ascorbyl palmitate, citric acid $_{\text {Phenoxyethanol }}$ & 0.10 \\
\hline
\end{tabular}

Note: All values are represented as percentages.

Clinical assessments were carried out at baseline (T0) and at the end of the trial (T30), and included the evaluation of scales and erythema in the case of psoriasis and the evaluation of itch and erythema in the case of eczema. Lesions and clinical manifestations were classified as absent (score 0 ), moderate (score 1), severe (score 2), or very severe (score 3).

Efficacy was statistically assessed through a procedure based on the individual's "permanence" or "migration state" (primary end point) depending on the entity of the score assigned to a symptom at T30 in comparison with the corresponding baseline score (T0). Thus, the "change of condition" generated the following categories of patients:

- In remission: when individuals with scores $>0$ at $\mathrm{T} 0$ have score $=0$ at $\mathrm{T} 30$;

- Improved: when individuals have lower scores at T30 than at $\mathrm{T} 0$;

- Unchanged: when individuals have the same scores at $\mathrm{T} 0$ and $\mathrm{T} 30$;

- Worsened: when individuals have higher scores at T30 than at $\mathrm{T} 0$.

We validated this statistical procedure in a previous study on the efficacy of $V$. myrtillus seed oil. ${ }^{20}$ As an internal control, we compared the effects of Bosexil ${ }^{\circledR}$ and V. myrtillus with the same placebo. The Psoriasis Area Severity Index (PASI) and the Eczema Area and Severity Index (EASI) were also evaluated (secondary end points).

Table 3 Composition of placebo

\begin{tabular}{ll}
\hline Aqua (water) & 83.40 \\
Glycerin & 2.00 \\
Disodium ethylenediaminetetraacetic acid & 0.10 \\
Imidazolidinyl urea & 0.30 \\
Polyacrylamide, CI3-I4 Isoparaffin, Laureth-7 & 3.00 \\
Hydrogenated polydecene & 8.50 \\
Caprylic/capric triglyceride & 2.00 \\
Lecithin, tocopherol, ascorbyl palmitate, citric acid & 0.10 \\
Phenoxyethanol & 0.60 \\
\hline
\end{tabular}

Note: All values are represented as percentages. 


\section{Statistical analysis}

Data were described by descriptive statistics. The comparison between groups was performed using Pearson's chi-square test; responses were evaluated with a Markov-like procedure. A $P$-value $<0.05$ was considered statistically significant.

\section{Results}

Twenty-six females and 33 men were considered in the study. Each group consisted of ten patients affected from psoriasis and ten patients with erythematous dermatitis (except the Bosexil $^{\circledR}$ group, in which patients with dermatitis numbered nine). At T0, all groups were similar in terms of severity of the disease. However, three patients in the placebo group, one in the V.myrtillus group, and two in the Bosexil ${ }^{\circledR}$ group suspended the treatment before T30 and were included with a score of 3 in the statistical analysis. No significant difference in terms of age or sex was observed among the three groups (Table 4).

\section{Change of condition}

The results from the "change of condition" (primary end point) analysis between placebo (group A) and two formulations (Bosexil ${ }^{\circledR}$ and V. myrtillus seed oil) are summarized in Tables 5 and 6.

\section{Psoriasis}

In patients with psoriasis (Table 5), signs of scales and erythema improved both with Bosexil ${ }^{\circledR}$ and with the V. myrtillus seed oil treatment in comparison with placebo. In particular, patients who received placebo had unchanged scales and erythema, and in $10 \%$ of cases worsened. Treatment with Bosexil ${ }^{\circledR}$ formulation improved scales (70\% of cases, $P=0.0001$ ) and erythema ( $60 \%$ of cases, $P=0.0281$ ) without any case of worsening. Treatment with $V$. myrtillus seed oil formulation improved erythema in $10 \%$ of cases, but $30 \%$ of patients were in remission at the end of therapy, therefore indicating that V. myrtillus seed oil treatment had positive effects on psoriatic erythema in $40 \%(P=0.253)$ of cases. In terms of scales, $V$. myrtillus seed oil improved the symptomatology in $80 \%$ of patients, and $20 \%$ was unchanged $(P=0.0003)$.

Table 4 Age of patients involved in the study (years)

\begin{tabular}{llll}
\hline Group & Disease & Female & Male \\
\hline Bosexil $^{\circledR}$ & Eczema & $27.3($ I3.0) & $31.5($ I 2.1$)$ \\
& Psoriasis & $4 I .0($ I6.6) & $51.5(12.2)$ \\
Vaccinium myrtillus & Eczema & $22.2(5.6)$ & $26.0(14.1)$ \\
seed oil & Psoriasis & $33.0(21.2)$ & $45.6(16.6)$ \\
Placebo & Eczema & $26.4(4.9)$ & $29.4($ II.8) \\
& Psoriasis & $40.3(15.4)$ & $38.2(9.2)$ \\
\hline
\end{tabular}

Note: Data are shown as mean (standard deviation).
Table 5 "Change of conditions" results from psoriasis patients group

\begin{tabular}{|c|c|c|c|}
\hline & \multicolumn{3}{|c|}{ Psoriasis group } \\
\hline & Bosexil $^{\circledR}$ & $\begin{array}{l}\text { Vaccinium } \\
\text { myrtillus seed oil }\end{array}$ & Placebo \\
\hline \multicolumn{4}{|l|}{ Erythema } \\
\hline In remission & 0 & 30 & 0 \\
\hline Improved & 60 & 10 & 0 \\
\hline Unchanged & 40 & 60 & 90 \\
\hline \multirow{2}{*}{ Worsened } & 0 & 0 & 10 \\
\hline & $P=0.028 \mathrm{I}$ & $P=0.253^{*}$ & \\
\hline \multicolumn{4}{|l|}{ Scales } \\
\hline In remission & 0 & 0 & 0 \\
\hline Improved & 70 & 80 & 0 \\
\hline Unchanged & 30 & 20 & 100 \\
\hline \multirow[t]{2}{*}{ Worsened } & 0 & 0 & 0 \\
\hline & $P=0.001$ & $P=0.0003$ & \\
\hline
\end{tabular}

Notes: $* P$-value is related to $40 \%$ positive response given by the addition in remission $(30 \%)+$ improved $(10 \%)$. All values are represented as percentages.

\section{Erythematous eczema}

In patients with erythematous eczema (Table 6), the Bosexil ${ }^{\circledR}$-containing formulation improved both itch $(60 \%$ of cases, $P=0.0115$ ) and erythema ( $60 \%$ of cases, $P=0.0115)$, without any case of worsening. V. myrtillus seed oil treatment improved itch and erythema in $66.7 \%(P=0.0020)$ and $77.8 \%$ $(P=0.0068)$ of patients, respectively. Patients on placebo showed unchanged or worsened itch and erythema.

\section{PASI and EASI}

The PASI and EASI scores observed in the three groups are reported in Tables 7 and 8, respectively. An improvement in both scores was observed with active treatments but not with placebo.

Figure 2 shows pictures of some psoriasis patients - one treated with Bosexil ${ }^{\circledR}$, one treated with V. myrtillus seed oil,

Table 6 "Change of conditions" results from erythematous eczema patients group

\begin{tabular}{llll}
\hline & \multicolumn{2}{l}{ Erythematous eczema } \\
\cline { 2 - 4 } & Bosexil $^{\circledR}$ & $\begin{array}{l}\text { Vaccinium } \\
\text { myrtillus seed oil }\end{array}$ & Placebo \\
\hline Itch & & 0 & 0 \\
In remission & 0 & 66.7 & 0 \\
Improved & 60 & 33.3 & 90 \\
Unchanged & 40 & 0 & 10 \\
Worsened & 0 & $P=0.0020$ & \\
Erythema & $P=0.0115$ & & 0 \\
In remission & 0 & 0 & 0 \\
Improved & 60 & 77.8 & 90 \\
Unchanged & 40 & 22.8 & 10 \\
Worsened & 0 & 0 & \\
\hline
\end{tabular}

Note: All values are represented as percentages. 
Table 7 Psoriasis Area Severity Index (PASI) score in psoriasis patients

\begin{tabular}{|c|c|c|c|c|c|c|}
\hline & \multicolumn{2}{|l|}{ Bosexil $^{\circledR}$} & \multicolumn{2}{|c|}{ Vaccinium myrtillus seed oil } & \multicolumn{2}{|l|}{ Placebo } \\
\hline & Baseline & End of study & Baseline & End of study & Baseline & End of study \\
\hline PASI & $14.5 \pm 3.2$ & $11.5 \pm 2.7^{*}$ & $16.9 \pm 3.7$ & $12.3 \pm 4.1 *$ & $15.1 \pm 3.0$ & $14.8 \pm 3.5$ \\
\hline
\end{tabular}

Note: $* P<0.05$ vs baseline.

Table 8 Eczema Area and Severity Index (EASI) score in eczema patients

\begin{tabular}{|c|c|c|c|c|c|c|}
\hline & \multicolumn{2}{|l|}{ Bosexil $^{\circledR}$} & \multicolumn{2}{|c|}{ Vaccinium myrtillus seed oil } & \multicolumn{2}{|l|}{ Placebo } \\
\hline & Baseline & End of study & Baseline & End of study & Baseline & End of study \\
\hline EASI & $21.0 \pm 7.3$ & $12.6 \pm 5.8^{*}$ & $20.6 \pm 6.3$ & $13.4 \pm 8.2 *$ & $20.8 \pm 9.4$ & $18.6 \pm 9.8$ \\
\hline
\end{tabular}

Note: $* P<0.05$ vs baseline.

\section{A Group Bosexil ${ }^{\odot}$}

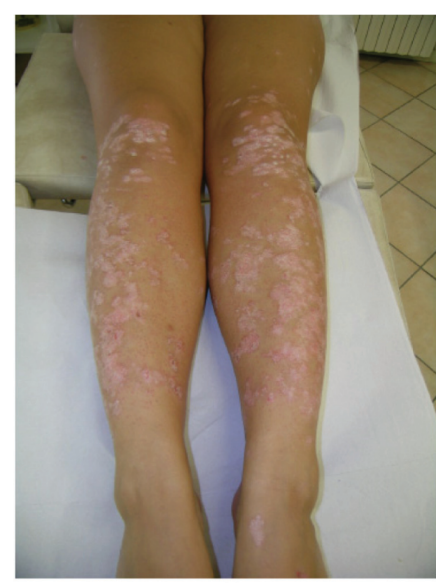

t0

\section{B Group Vaccinum myrtillus seed oil}

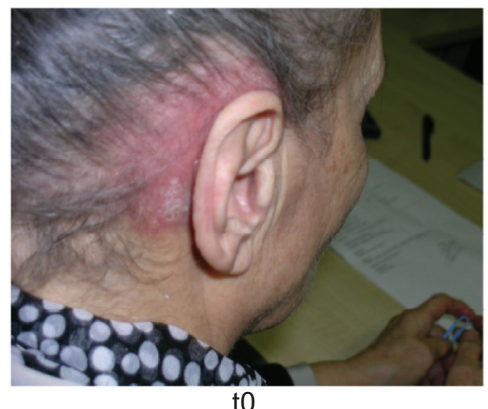

\section{Group placebo}

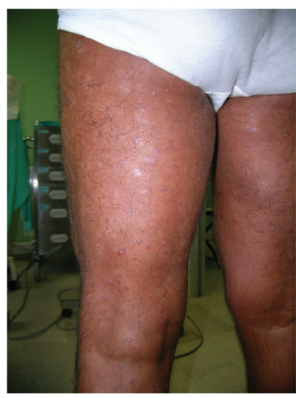

t0

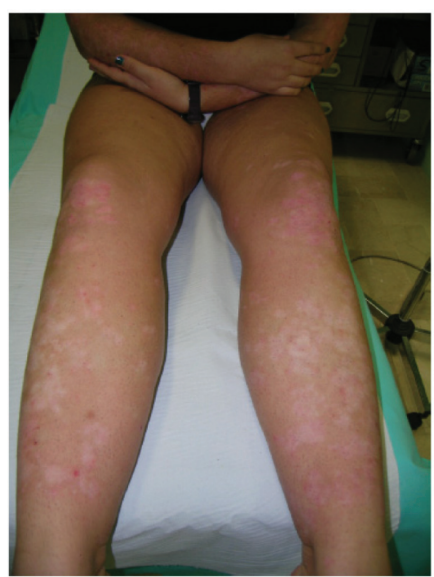

t1

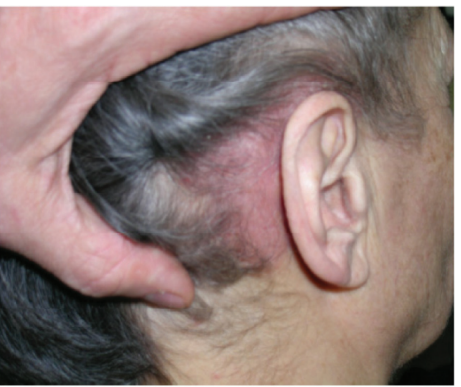

t1

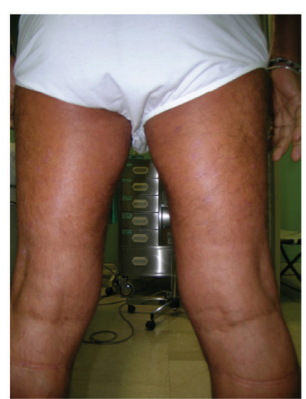

t1

Figure 2 Pictures of some psoriasis patients - one treated with Bosexil ${ }^{\circledR}(\mathbf{A})$, one treated with Vaccinum myrtillus seed oil (B), and the other assigned to placebo (C). Pictures were taken at baseline $(\mathrm{t} 0)$ and at the end of the study $(\mathrm{t} \mathrm{l})$. 
and the other assigned to placebo. The pictures were taken at baseline and at the end of the study.

\section{Discussion}

This study aimed to compare, for the first time to our knowledge, the efficacy of topical preparation of Bosexil ${ }^{\circledR}$ and $V$. myrtillus seed oil vs a placebo in individuals with a diagnosis of psoriasis or erythematous dermatitis. Indeed, these formulations are particularly rich in polyunsatured fatty acids ( $V$. myrtillus seed oil) or BAs (Bosexil $\left.{ }^{\circledR}\right)$ that exert anti-inflammatory effects, thus may provide benefits in skin inflammatory diseases. BAs in Bosexil ${ }^{\circledR}$ formulation were more active in the treatment of psoriatic scales than erythema, and were similarly efficient to treat itch and erythema in patients affected by eczema. This formulation was designed according to the Phytosome $^{\circledR}$ delivery system ${ }^{21}$ in order to improve the bioavailability and skin affinity of BAs. Consistently with previous results, ${ }^{20}$ this study confirmed the efficacy of $V$. myrtillus seed oil preparation in the treatment of major symptoms in both psoriasis and eczema, such as erythema and scales and erythema and itch, respectively. Noteworthy is that V. myrtillus seed oil was associated with three remissions in the treatment of erythema in psoriatic patients. Bilberry fruits contain small seeds rich in alpha-linolenic acid and linoleic acid, which are considered essential nutrients (the human body, in fact, lacks the necessary enzymes to introduce an unsaturation beyond position omega-9). Linoleic acid and alpha-linolenic acid are involved in the skin barrier function. ${ }^{22}$

One issue in the management of chronic diseases such as psoriasis is the adherence to treatment. Few data are available in literature, but the most frequently mentioned reasons for nonadherence to topical treatment are low efficacy, time consumption, and poor cosmetic characteristics of topical agents. ${ }^{23}$ This study has tested a twice-daily administration which determined an effective improvement of disease. However, the study lasted a short period of time and included an overall limited number of patients; therefore, long-term, larger studies should be recommended to assess the adherence to this protocol in real life. Moreover, earlier assessments (eg, at day 15) could have been performed to collect information on the early efficacy of both Bosexil ${ }^{\circledR}$ and $V$. myrtillus seed oil. Last, other end points (eg, patient-related severity score or the Dermatology Life Quality Index) have not been evaluated in this study.

In addition to the anti-inflammatory/barrier-protecting effects of polyunsatured fatty acids or BAs, a cosmeceutical formulation of these creams may provide the adjunctive benefits that can restore and protect the skin barrier function, increase the remission times between flare-ups, and enhance the pharmaceutical effects of active compounds. ${ }^{24}$

\section{Conclusion}

In conclusion, BAs contained in Bosexil ${ }^{\circledR}$ and formulated according to the Phytosome ${ }^{\circledR}$ delivery system ${ }^{21}$ may be eligible components for the formulation of preparation with anti-inflammatory properties for the treatment of psoriatic and eczematous symptoms. We speculate that this formulation could also be applied to other inflammatory skin disorders, including contact, atopic, seborrheic, nummular, chronic palmoplantar, and generalized exfoliative or chronic lichen simplex dermatitis.

\section{Acknowledgments}

Editorial assistance for the preparation of this manuscript was provided by Luca Giacomelli $\mathrm{PhD}$ and Elisa Sala PhD; this assistance was funded by Indena S.p.A.

\section{Disclosure}

GM and ST are employees of Indena S.p.A., the manufacturer of the products used in this study. The authors report no other conflicts of interest in this work.

\section{References}

1. Sohn A, Frankel A, Patel RV, Goldenberg G. Eczema. Mt Sinai J Med. 2011;78(5):730-739.

2. Langner MD, Maibach HI. Pruritus measurement and treatment. Clin Exp Dermatol. 2009;34(3):285-288.

3. Schon MP, Boehncke WH. Psoriasis. N Engl J Med. 2005;352(18): 1899-1912.

4. Schon MP, Boehncke WH, Brocker EB. Psoriasis: clinical manifestations, pathogenesis and therapeutic perspectives. Discov Med. 2005;5(27):253-258.

5. Schon MP, Ludwig RJ. Lymphocyte trafficking to inflamed skin molecular mechanisms and implications for therapeutic target molecules. Expert Opin Ther Targets. 2005;9(2):225-243.

6. Belge K, Bruck J, Ghoreschi K. Advances in treating psoriasis. F1000Prime Rep. 2014;6:4.

7. Papoutsaki M, Costanzo A. Treatment of psoriasis and psoriatic arthritis. BioDrugs. 2013;27 Suppl 1:3-12.

8. Samarasekera EJ, Sawyer L, Wonderling D, Tucker R, Smith CH. Topical therapies for the treatment of plaque psoriasis: systematic review and network meta-analyses. Br J Dermatol. 2013;168(5):954-967.

9. Mustonen A, Mattila K, Leino M, Koulu L, Tuominen R. The costs of psoriasis medications. Dermatol Ther (Heidelb). 2013;3(2):169-177.

10. Poeckel D, Werz O. Boswellic acids: biological actions and molecular targets. Curr Med Chem. 2006;13(28):3359-3369.

11. Wang Q, Pan X, Wong HH, et al. Oral and topical boswellic acid attenuates mouse osteoarthritis. Osteoarthritis Cartilage. 2014;22(1): $128-132$.

12. Singh S, Khajuria A, Taneja SC, Johri RK, Singh J, Qazi GN. Boswellic acids: a leukotriene inhibitor also effective through topical application in inflammatory disorders. Phytomedicine. 2008;15(6-7):400-407.

13. Borrelli F, Capasso F, Capasso R, et al. Effect of Boswellia serrata on intestinal motility in rodents: inhibition of diarrhoea without constipation. Br J Pharmacol. 2006;148(4):553-560. 
14. Ammon HP. Modulation of the immune system by Boswellia serrata extracts and boswellic acids. Phytomedicine. 2010;17(11):862-867.

15. Abdel-Tawab M, Werz O, Schubert-Zsilavecz M. Boswellia serrata: an overall assessment of in vitro, preclinical, pharmacokinetic and clinical data. Clin Pharmacokinet. 2011;50(6):349-369.

16. Altmann A, Poeckel D, Fischer L, Schubert-Zsilavecz M, Steinhilber D, Werz O. Coupling of boswellic acid-induced $\mathrm{Ca} 2+$ mobilisation and MAPK activation to lipid metabolism and peroxide formation in human leucocytes. Br J Pharmacol. 2004;141(2):223-232.

17. Ammon HP. Boswellic acids in chronic inflammatory diseases. Planta Med. 2006;72(12):1100-1116.

18. Syrovets T, Buchele B, Krauss C, Laumonnier Y, Simmet T. Acetyl-boswellic acids inhibit lipopolysaccharide-mediated TNF-alpha induction in monocytes by direct interaction with IkappaB kinases J Immunol. 2005;174(1):498-506.

19. Wang H, Syrovets T, Kess D, et al. Targeting NF-kappa B with a natural triterpenoid alleviates skin inflammation in a mouse model of psoriasis. J Immunol. 2009;183(7):4755-4763.
20. Maramaldi G, Togni S, Meneghin M, Appendino G, Biondi M, Di Pierro F. Olio di semi di mirtillo. Un cosmeceutico nel trattameto di soggetti con cute eczematosa o psoriasica. Cosmotech. 2013.

21. Semalty A, Semalty M, Rawat MS, Franceschi F. Supramolecular phospholipids-polyphenolics interactions: the PHYTOSOME strategy to improve the bioavailability of phytochemicals. Fitoterapia. 2010;81(5):306-314.

22. Simopoulos AP. The importance of the ratio of omega-6/omega-3 essential fatty acids. Biomed Pharmacother. 2002;56(8):365-379.

23. Devaux S, Castela A, Archier E, et al. Adherence to topical treatment in psoriasis: a systematic literature review. JEur Acad Dermatol Venereol. 2012;26 Suppl 3:61-67.

24. Luger T, Seite S, Humbert P, Krutmann J, Triller R, Dreno B. Recommendations for adjunctive basic skin care in patients with psoriasis. Eur J Dermatol. 2014;24(2):194-200.
Clinical, Cosmetic and Investigational Dermatology

\section{Publish your work in this journal}

Clinical, Cosmetic and Investigational Dermatology is an international, peer-reviewed, open access, online journal that focuses on the latest clinical and experimental research in all aspects of skin disease and cosmetic interventions. All areas of dermatology will be covered; contributions will be welcomed from all clinicians and

\section{Dovepress}

basic science researchers globally. This journal is indexed on CAS The manuscript management system is completely online and includes a very quick and fair peer-review system, which is all easy to use. Visit http://www.dovepress.com/testimonials.php to read real quotes from published authors.

Submit your manuscript here: http://www.dovepress.com/clinical-cosmetic-and-investigational-dermatology-journal 